\title{
Towards Location Based QoS-Aware Network Selection Mechanism for the Nomadic Mobile Services
}

\author{
Pravin Pawar ${ }^{1}$, Bert-Jan van Beijnum ${ }^{1}$, Katarzyna $\mathrm{Wac}^{1,2}$, Hermie Hermens ${ }^{1}$, Dimitri Konstantas ${ }^{2}$ \\ ${ }^{I}$ Faculty of Electrical Engineering, Mathematics and Computer Science \\ Department of Computer Science \\ University of Twente, The Netherlands. \\ \{p.pawar,beijnum,wac\}@utwente.nl \\ h.hermens@rrd.nl \\ ${ }^{2}$ Advanced Systems Group, Centre Universitaire d'Informatique \\ University of Geneva, Switzerland. \\ \{dimitri.konstantas, katarzyna.wac\}@cui.unige.ch
}

\begin{abstract}
The advances in the area of location based computing will soon make it feasible to predict the availability of wireless networks and their application level Quality of Service (QoS) characteristics along the location and time dimensions. Such predictions are referred to as QoS predictions which are provided by the QoS Context Source (QoSCS) hosted in the fixed network. Herewith we present a network selection mechanism on the mobile device which requests QoS predictions as needed and uses these predictions combined with the current location and time to select the wireless network which satisfies QoS requirements of the services hosted on a mobile device. We evaluate the performance of the proposed scheme using simulations and compare the results with the other mechanism that only uses information locally available on the mobile device for the network selection. The results obtained from the simulations encourage further system development and prototyping.
\end{abstract}

\section{Introduction}

Availability of a wide range of multi-homed mobile devices capable of connecting to the Internet using multiple Network Interfaces (NI) combined with the auxiliary hardware (e.g. GPS receiver), smart software (e.g. NetPerf) and increasing user willingness to share the information available on and around mobile device (e.g. location, network information), are capable of generating a vast amount of experienced Quality of Service (QoS) information. One of the recent research works in the area of location based computing is to use such information collected over a period of time to predict the availability of the wireless networks and their application level QoS characteristics (throughput and delay) along the location and time dimensions (so called $Q o S$ predictions) [1]. Similar to the use of geographic maps in the GPS-based navigation systems, we foresee that the map of QoS predictions could be very helpful for the applications sending (receiving) data from (on) a mobile device and it could help a mobile device to navigate (i.e. network selection) through an area which is covered by multiple wireless networks. The QoS predictions are provided by an entity called as QoS Context Source (QoSCS); a service hosted in the fixed network.

Our application for the work reported herewith is in the mobile (M)-Health domain for the remote patient monitoring service [2] where a patient's mobile device acquires the vital signs data from sensors attached to the patient's body, (pre-) processes the data locally at the mobile device, and sends the data in real-time using wireless connectivity to the back-end system in the fixed network. In line with the commercially available handheld mobile devices, we consider that a mobile device is equipped with one Wireless Wide Area Network (WWAN) interface (e.g. GPRS and/or UMTS) and one Wireless Local Area Network (WLAN) interface (e.g. WiFi). In the existing implementation [3], for the real-time data transfer, the remote patient monitoring system always prefers WLAN network over the WWAN network. In [3], the WLAN network is selected randomly in case of the availability of multiple WLAN networks. We refer to this network selection mechanism as BASIC.

In this paper, we extend the work reported in [3]. More specifically; we design and develop contextaware network selection mechanism (to be referred to as $A D V A N C E D$ ) which uses QoS predictions combined with the current location and time to select the optimal 


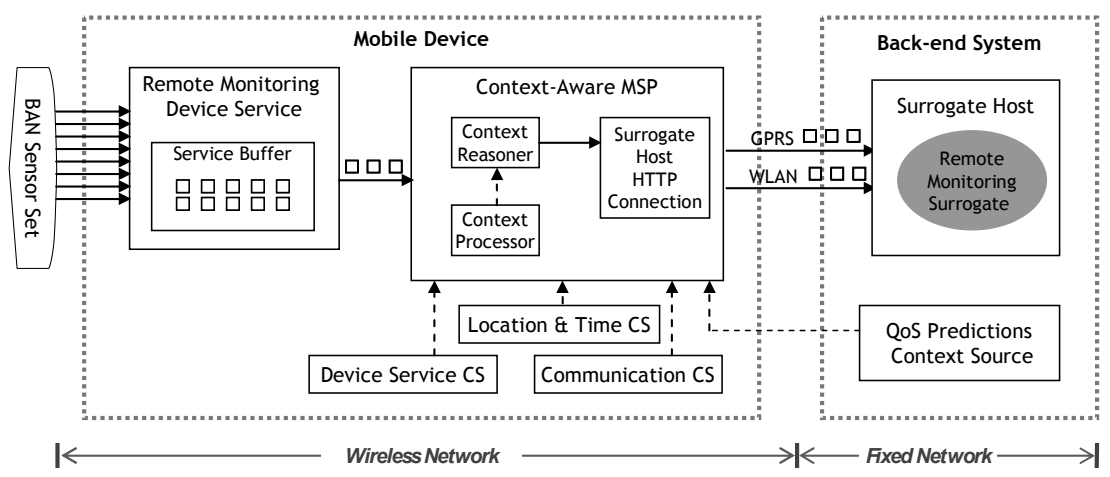

Figure 1: Elements of Remote Patient Monitoring System

network which satisfies the QoS requirements of the remote patient monitoring service.

However, the QoSCS is still in the development phase [1]. Hence to get a better understanding of how the ADVANCED scheme could be viable in practice, we performed a series of simulations. To this end, we built the QoSCS simulator, a user trip simulator and interaction mechanism between the remote patient monitoring service and QoSCS.

The reminder of this paper is as follows. Section 2 introduces the elements of the remote patient monitoring system. Section 3 briefly presents the metrics used for comparing BASIC and ADVANCED schemes. Section 4 is on the simulation setup. Section 5 presents the simulation results. Section 6 describes the related work. Section 7 concludes the paper and provides the future work areas.

\section{Remote Patient Monitoring System}

The elements of the remote patient monitoring system are shown in the Figure 1. The elements of this system are distributed on the mobile device and the Back-end system hosted in the fixed network. The description of these elements is as follows:

Body Area Network (BAN) Sensor set: A BAN sensor set processes vital signs measured by the sensors attached to the patient's body, and outputs multiple channels of the patient vital signs data.

Remote Monitoring Service: This service consists of two components: 1) Remote Monitoring Device Service on the mobile device; and 2) Remote Monitoring Surrogate hosted by the Surrogate Host in the fixed network. The monitoring device service consists of a service buffer which maintains the number of packets waiting to be processed by the Context-Aware Mobile Service Platform (explained in the following). This number is mapped to the fill level $(0-100)$ of this buffer.

Context-Aware Mobile Service Platform: Mobile Service Platform (MSP) [3] is a central component responsible for the development, deployment and the lifecycle management of the remote patient monitoring system. MSP uses Surrogate Host HTTP Connection (streaming data delivery using HTTP protocol) for communication between the mobile device and back- end system. The Context-Aware MSP (CA-MSP) uses context information obtained from a number of context sources (explained in the following) and subsequent context changes for the dynamic selection of and handover to the optimal wireless network which satisfies the following network selection objectives: 1) Maximize remote monitoring service's bandwidth requirements; 2) Minimize remote monitoring service's delay requirements. For this purpose, MSP interacts with the Context Sources (CS) shown in the Table 1.

Table 1: Description of Context Sources

\begin{tabular}{|l|l|}
\hline \multicolumn{1}{|c|}{ CS Name } & \multicolumn{1}{|c|}{ Context Information } \\
\hline $\begin{array}{l}\text { Location } \\
\text { And Time } \\
\text { Context Source }\end{array}$ & $\begin{array}{l}\text { Coordinates of the device's current } \\
\text { geographic location (longitude, } \\
\text { latitude) and time (Date, } \\
\text { HH:MM:SS as obtained from the GPS } \\
\text { receiver. }\end{array}$ \\
\hline $\begin{array}{l}\text { Communication } \\
\text { Context Source } \\
{[4]}\end{array}$ & $\begin{array}{l}\text { A list of mobile networks along with provider } \\
\text { names, technologies, theoretical uplink } \\
\text { throughput and delay (Network Cross } \\
\text { Layer Info. in XML) in the } \\
\text { surroundings of a mobile device at a given } \\
\text { time and location, current state of the } \\
\text { network interfaces on the mobile device. }\end{array}$ \\
\hline $\begin{array}{l}\text { Device Service } \\
\text { Context Source }\end{array}$ & $\begin{array}{l}\text { Required bandwidth and delay of remote } \\
\text { monitoring device service. }\end{array}$ \\
\hline $\begin{array}{l}\text { QoS- } \\
\text { Predictions }\end{array}$ & $\begin{array}{l}\text { All the available wireless networks as } \\
\text { specified by provider names, network names } \\
\text { and technologies along with their coverage } \\
\text { (QoSCS) [1] } \\
\text { ranges and availability at a given } \\
\text { location/time and predicted QoS information } \\
\text { (bandwidth and delay). }\end{array}$ \\
\hline
\end{tabular}

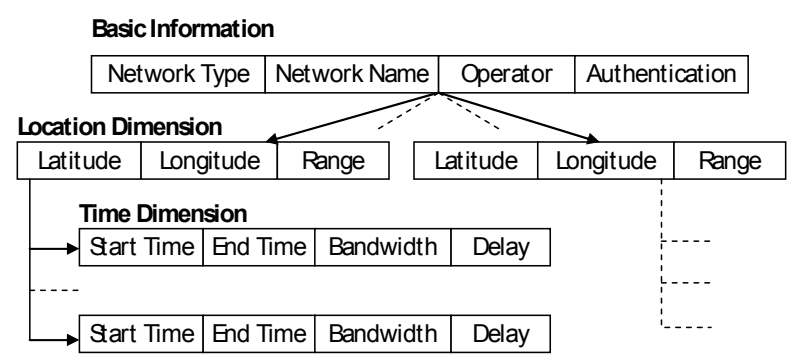

Figure 2. Structure of QoS Predictions

The QoS predictions provided by the QoSCS for a particular wireless network typically contains one element providing basic information such as type, network name, operator name, authentication type (e.g. WiFi, Guest WLAN, University of 
Geneva, and Open), multiple elements providing location-dimension information such as longitude, latitude, range (e.g. 46.179956, 6.13896, 55m) and for each such element, multiple elements providing time-dimension information such as start time, end time, offered bandwidth, offered delay (e.g. 24 APR 2008 13:55:30，24 APR 2008 14:20:33， 15149 bps, $650 \mathrm{~ms}$ ) as shown in the Figure 2.

The role of the Context Processor (CP) component is to get/subscribe context information from the context sources and provide a necessary aggregated context information to the Context Reasoner (CR) to be able to make a network selection decision at a given location and time. Upon the activation of the remote monitoring device service, $\mathrm{CP}$ obtains the current location and time from the location and time $\mathrm{CS}$ and provides this information to the QoSCS to obtain QoS-predictions. After conducting a set of experiments, in the current setting, QoSCS sends predictions for a pre-defined number of networks (e.g. 30 networks) closest to the current location of the mobile device. Along with the QoS predictions, the QoSCS specifies the distance between the farthest network from the current location of mobile device (predictions range) and the time for which the QoS-predictions are valid (predictions time).

$\mathrm{CP}$ takes care of requesting fresh predictions when the user is on the boundary (within certain distance) of the predictions range or the predictions time is about to expire. The QoS predictions useful at the current location and time (i.e. the wireless networks to which a mobile device can connect immediately) combined with the QoS requirements of the remote monitoring service together form the current context snapshot which is later sent to $\mathrm{CR}$ for further processing. Based on the subsequent context changes (e.g. location change, time change, service QoS requirements change, network unavailable) CP takes an appropriate action such as create current context snapshot, update QoS requirements or request (new) QoS predictions.

Based on the information received from the current context snapshot, CR applies Analytic Hierarchy Process (AHP) based algorithm to optimize the network selection objectives using the following AHP steps: 1) Decide the relative importance of the optimization objectives; 2) Compute relative weight of each available network for each objective by considering the QoS requirements of the remote monitoring service; 3) Calculate overall score for each network and select the network having the highest score for the handover.

\section{Performance Metrics for Comparison}

Herewith we claim that the ADVANCED scheme which relies on the location and time based QoS predictions for the network selection provides superior
QoS experience to the remote patient monitoring service as compared to the BASIC scheme which randomly selects WLAN network for the data transfer. To substantiate this claim; we came up with the following performance comparison metrics:

Average data sent: Average amount of data sent from the mobile device using specific network selection scheme.

Average buffer fill level: Average amount of data accumulated in the monitoring device service buffer waiting for its transmission by CA-MSP.

Average data loss: Average amount of data lost over the simulation period. Data loss occurs only when the service buffer is full (i.e. can't hold more data.)

Average delay: Average of delay experienced by the remote monitoring device service by using the selected network over the simulation period.

Disconnection time: The time for which there is no network connectivity available to the mobile device. Note that disconnection occurs in the following cases: 1) Mobile devices moves out of the coverage area of WLAN network; 2) During the handover start and handover complete process.

Number of handovers: The number of handovers experienced by the BASIC and ADVANCED network selection mechanisms.

\section{Simulation Environment}

For the purpose of conducting simulations involving QoSCS, user mobility and BASIC and ADVANCED network selection schemes, we built QoSCS simulator and user trip simulator. In contrast to the traditional simulation topology (e.g. square plane areas with uniformly placed WLAN base stations [5]) for the network selection mechanisms, the QoSCS simulator takes a close-to-real life approach. Nowadays most of the businesses and institutes are covered by WLAN. Moreover, the accurate location listings of businesses and institutes are available on the Internet (in our case, Google maps server). Based on these two facts, QoSCS simulator uses the locations of the businesses and institutes to assign WLAN base stations. Similar to the approach used by the QoSCS simulator, the user trip simulator also uses the route between source and destination coordinates information available on the Internet (in our case, Google maps server) to model user movements between a source and destination locations.

The simulation environment considers two types of wireless networks namely WiFi (WLAN) and GPRS (WWAN). The QoSCS simulator obtains the list of around 2000 businesses from the Google maps server approximately covering area of radius $70 \mathrm{~km}$ around Geneva (Switzerland) city centre and WiFi base stations are assigned to these locations. For simulating 

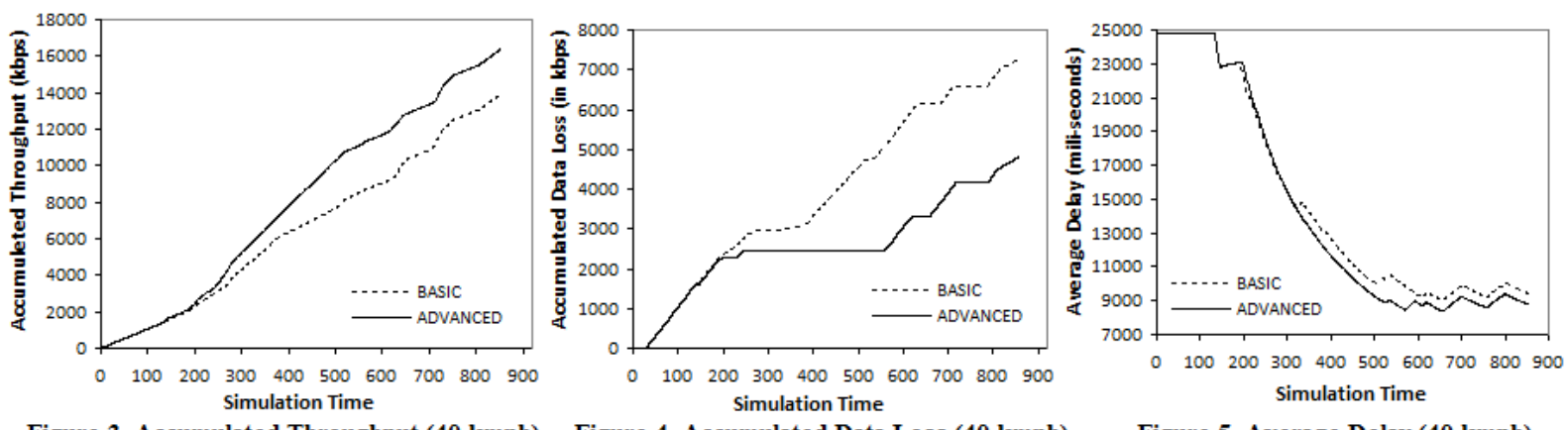

Figure 3. Accumulated Throughput (40 kmph)

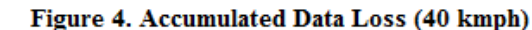

Figure 5. Average Delay (40 kmph)
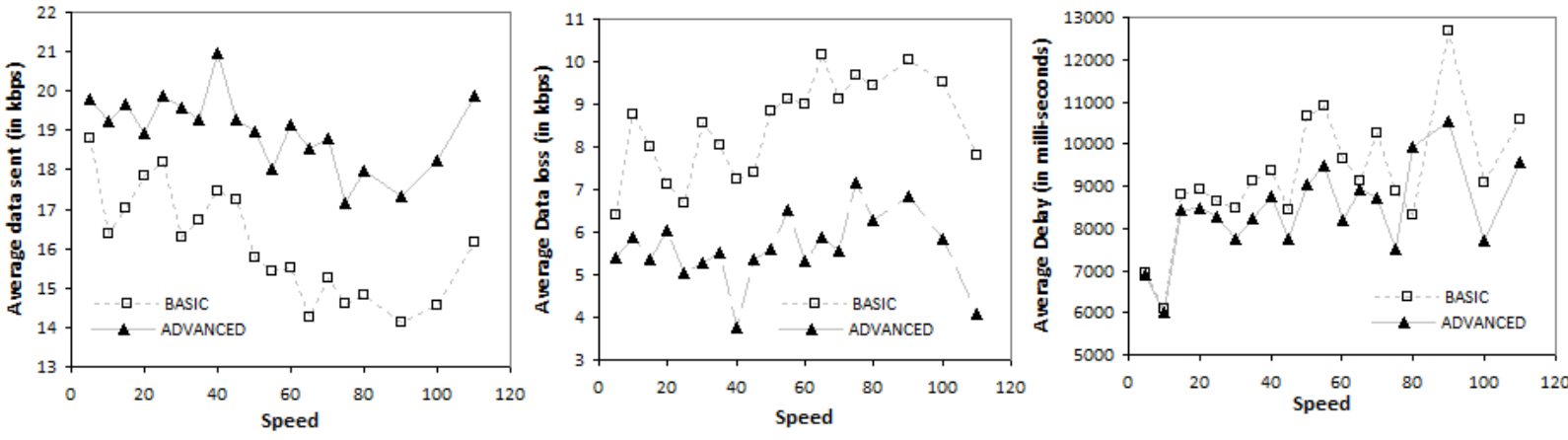

Figure 6. Average Data Sent vs. Speed

Figure 7. Average Data Loss vs. Speed

Figure 8. Average Delay vs. Speed
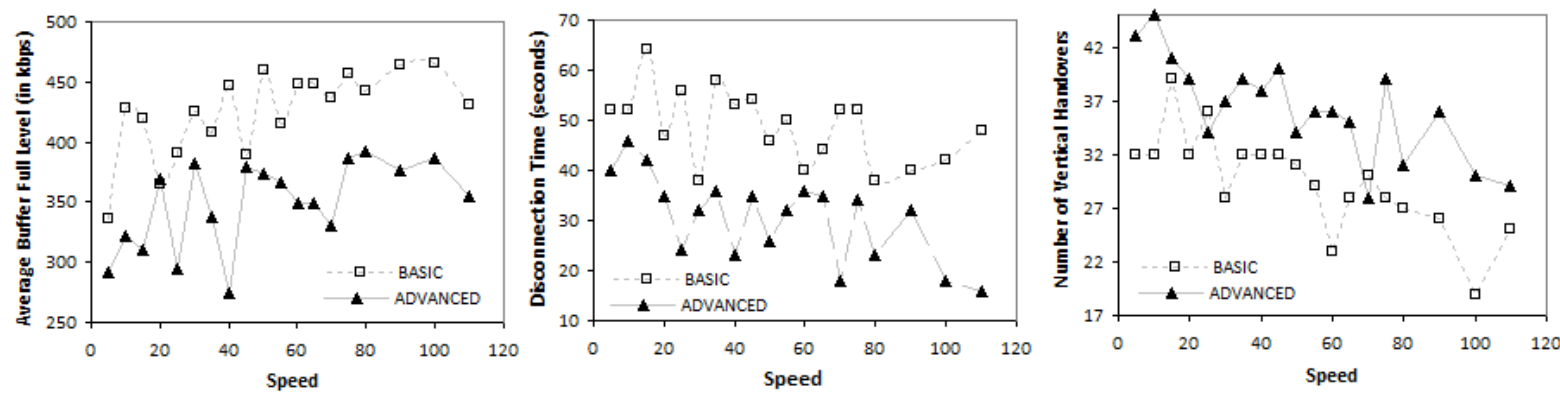

Figure 9. Average Buffer Fill LEvel vs. Speed

Figure 10. Disconnection Time vs. Speed

Figure 11. Number of Handovers vs. Speed

user mobility, the trip originates at Carouge and ends at Vernier (both are municipalities in the Canton of Geneva) via Geneva city centre covering a distance of $8.470 \mathrm{~km}$ and consisting of 107 sub-steps (transition from one step to another roughly corresponds to the direction change on the user mobility path). The simulation is conducted for a variety of user speeds $(5$, $10,15,20,25,30,35,40,45,50,55,60,65,70,75$, $80,90,100$ and $110 \mathrm{~km} / \mathrm{h})$.

The other simulation parameters and their values are shown in the Table 2 . The bandwidth and delay requirements of the remote patient monitoring device service are $25580 \mathrm{bps}$ and $500 \mathrm{msec}$ respectively [3]. The maximum radius of the $\mathrm{WiFi}$ base station based on the claim in [6]. As a reasonable assumption, GPRS network is available wherever user goes. The QoScharacteristics of both, GPRS and WLAN networks change according to the location and time.

\section{Simulation results}

The accumulated throughput, accumulated data loss and average delay respectively during the simulation run where the user moves at the speed of 40 $\mathrm{km} / \mathrm{h}$ are shown in the Figure 3, Figure 4 and Figure 5 respectively. As can be observed from these graphs, the ADVANCED scheme outperforms the BASIC scheme. A similar trend is observed for the simulation with other speed values.

The graphs in the Figure 6, Figure 7, Figure 8 and Figure 9 show the average data sent, average data lost, average delay and average buffer fill level vs. speed respectively for the BASIC and ADVANCED schemes. As can be observed from these figures, BASIC scheme performs worse compared to ADVANCED scheme.

The graphs in the Figure 10 and Figure 11 depict the disconnection time (in seconds) and number of vertical handovers respectively for the BASIC and ADVANCED schemes. On an average, BASIC scheme results in larger number of disconnections as compared to the ADVANCED scheme. We observed that the 
BASIC scheme results in the lesser number of handovers as compared to the ADVANCED scheme. This due to the reason that BASIC scheme randomly connects to the WLAN network and the vertical handover is done only when the mobile device is out of WLAN network range. In contrast, the ADVANCED tries to optimize the network selection based on the remote monitoring service requirements.

Table 2: Simulation Parameters

\begin{tabular}{|l|l|l|}
\hline \multicolumn{1}{|c|}{ Simulation Parameter } & \multicolumn{1}{c|}{ WLAN } & \multicolumn{1}{c|}{ GPRS } \\
\hline Maximum bandwidth & $55000 \mathrm{bps}$ & $25000 \mathrm{bps}$ \\
\hline Minimum bandwidth & $10000 \mathrm{bps}$ & $10000 \mathrm{bps}$ \\
\hline Maximum delay & $1000 \mathrm{~ms}$ & $25000 \mathrm{~ms}$ \\
\hline Minimum delay & $35 \mathrm{~ms}$ & $1000 \mathrm{~ms}$ \\
\hline Maximum coverage radius & 300 meters & Always available \\
\hline Minimum coverage radius & 10 meters & Always available \\
\hline
\end{tabular}

Note that the results obtained from the simulations do not result in smooth graphs. This is because of randomness (in certain limits) in the QoS CS simulator to generate variable QoS predictions over a period of time. However, from the simulation results, the following general trend is clear: The ADVANCED scheme which uses location and time based QoS predictions information available in the fixed network to select the optimal network outperforms the BASIC scheme which relies only on the information available on the mobile device.

\section{Related Work}

Considering the scope of our work reported in this paper, we focus on the work which takes into account the QoS requirements of the mobile applications to take a network selection decision. Balasubramanian and Indulska [7] propose a vertical handover mechanism that matches QoS requirements of multimedia applications onto the location based QoS provided by the wireless networks. In [8] Hong et. al. propose combining QoS requirements of all the mobile applications and handover to the suitable network. It uses dwelling timer for avoiding ping-pong effect. The scheme in [8] is evaluated for its potential to reduce the number of unnecessary handovers and application failures. Chen et al. [5] propose to use a Location Service Server (LSS) in the fixed network which provides information such as coverage area, throughput and latency of available wireless networks around a mobile device. QoSCS is similar to LSS, except that QoSCS is designed to provide QoS predictions along a user trip path in addition to the above information.

In comparison to the related work described herewith, the distinguishing aspects of our work reported herewith are as follows: 1) Design and development of the ADVANCED network selection mechanism which uses location and time QoS predictions available in the fixed network; 2) Use of the geographic information available on the Internet to build QoSCS simulator and to realistically model user movements along the user trip path; 3) Extensive simulations to compare the performance of the ADVANCED scheme with the BASIC scheme.

\section{Conclusion}

Owing to the recent developments in the area of location based computing, mobile devices in the near future will increasingly exploit the location and time based QoS predictions available in the fixed network to provide better QoS experience to the hosted services. Herewith we compare two network selection schemes; namely ADVANCED scheme which uses such QoS predictions and BASIC scheme which selects the wireless network based on the information available locally on the mobile device. From the extensive simulation experiments for the remote patient monitoring service, we conclude that the ADVANCED scheme provides a superior QoS experience to the mobile services.

\section{Acknowledgements}

This work is supported by Dutch Freeband Awareness project (under grant BSIK5902390).

\section{References}

[1] Wac K., et. al., "QoS-predictions service: infrastructural support for proactive QoS- and context-aware mobile services". CAMS Workshop, On The Move 2006, OctNov. 2006, Montpellier, France,.

[2] van Halteren, A., Bults, R., et al., "Mobile Patient Monitoring: The MobiHealth System". The Journal on Information Technology in Healthcare, 2(5), 2004.

[3] Pawar, P., van Beijnum, B. J., Wac, K., Maret, P., et. al., "Performance evaluation of the context-aware handover mechanism for the nomadic mobile services in remote patient monitoring", Elsevier Computer Communications, (August 2008), doi:10.1016/j.comcom.2008.04.020.

[4] Peddemors, A., H. Eertink, and I. Niemegeers., "Communication Context for Adaptive Mobile Applications." In 3rd IEEE International Conference on Pervasive Computing and Communications Workshops (PERCOMW05), March 2005, Hawaii, USA.

[5] Chen, W.-T. et. al., "Active Application Oriented Vertical Handoff in Next-Generation Wireless Networks", WCNC 2005. LA, USA.

[6] Vivato Technical White Paper, "Metropolitan Wireless LAN/Man Deployment". June 2004, http://www.vivato.net, Accessed: 24 July, 2008.

[7] Balasubramaniam, S. and J. Indulska, "Vertical handover supporting pervasive computing in future wireless networks", Elsevier Computer Communications, 27(8): p. 708-719, March 2004.

[8] Hong, C. P., Kang, T. H., and Kim, S. D., "A Profile Based Vertical Handoff Scheme for Ubiquitous Computing Environment", Asia-Pacific Network Operations and Management Symposium 2006, September 2006, Busan, Korea. 